Worringen, U., \& Muschalla, B. (2017). Empfehlungen für psychologische Interventionen in der rheumatologischen Rehabilitation. Aktuelle Rheumatologie, 42, 137-142.

\title{
Empfehlungen für psychologische Interventionen in der rheumatologischen Rehabilitation
}

\author{
U.Worringen, B. Muschalla
}

\section{Korrespondenzadresse:}

Dr. Ulrike Worringen

DRV Bund, Abteilung Rehabilitation

Hohenzollerndamm 47

10713 Berlin

dr.ulrike.worringen@drv-bund.de 


\section{Zusammenfassung}

Psychologische Interventionen stellen einen wichtigen Bestandteil der medizinischen Rehabilitation der Deutschen Rentenversicherung dar. Sie zielen bei erwerbsfähigen Rehabilitanden ebenso wie alle anderen therapeutischen Leistungen auf die Befähigung zur Teilhabe am Erwerbsleben ab. Bei rheumatologischen Erkrankungen werden von psychologischer Seite insbesondere die Themen der Krankheits- und Schmerzbewältigung in Einzel- und Gruppengesprächen aufgegriffen. Mit der Krankheit einhergehende chronische Schmerzen oder auch Folgeerscheinungen der medikamentösen Therapie sind dabei nicht selten gepaart mit einer geringeren Lebensqualität und einer eingeschränkten Teilhabe am gesellschaftlichen Leben. Zudem können Ängste und depressive Stimmungen die rheumatische Erkrankung begleiten. In diesem Kontext eine behandlungsbedürftige psychische Erkrankung von krankheitsbedingtem Leiden abzugrenzen und ggf. eine fachgerechte Unterstützung einzuleiten stellt eine Herausforderung in der medizinischen Rehabilitation dar.

Edukative Gruppenangebote können problem- und krankheitsbezogen durchgeführt werden und befördern den Austausch mit Gleichbetroffenen. Im Rahmen von krankheitsspezifischen Patientenschulungen steht die Vermittlung von krankheitsbezogenem Wissen und krankheitsbezogenen Kompetenzen im Mittelpunkt. Hierbei ist die enge fachliche Zusammenarbeit aller therapeutischen Berufsgruppen der medizinischen Rehabilitation gefragt. Bei Rehabilitanden mit besonderen beruflichen Problemlagen können psychologische Stressbewältigungs-Gruppen oder andere arbeitsbezogene Gruppenangebote das Gesamtspektrum der beruflich orientierten Therapieleistungen ergänzen. Über die Behandlungsmaßnahmen hinaus können Psychologen und Psychotherapeuten in der medizinischen Rehabilitation mit einer verhaltens- und fähigkeitsorientierten Befundung einen wichtigen Beitrag zur sozialmedizinischen Leistungsbeurteilung leisten.

Schlüsselwörter: Psychologische Behandlung, Krankheitsmanagement, Rheumatologie, Rehabilitation 


\begin{abstract}
Psychological interventions are an important aspect in medical rehabilitation of Federal German Pension Fund. Their aim is - similar to other rehabilitation treatment aspects - to improve work ability over the long-term course and prevent disability. In rheumatologic illness, management of illness- and pain can be focused in psychological interventions. Chronic pain or long-term side effects of medical therapy are often coming along with reduced quality of life and reduced participation in general life. Furthermore, anxiety and depressive symptoms may occur.

Identifying a clinically relevant mental disorder, and distinguish normal suffering from mental disorder is important in somatic rehabilitation and needs specialists in mental health clinical diagnostic. Patient education is mostly done in groups, which focus on problem solving and illness management. Structured patient education programs should be done using all professionals from the rehabilitation team, e.g. physicians, psychologists, sport therapists, social workers or others in cooperation.

In patients with additional vocational problems, stress management and work-directed conflict management education may be done. Additionally, psychologists and psychotherapists' expertise of behavior observation and behavior description should be used for the medical work ability description in rehabilitation reports.
\end{abstract}

Keywords: psychological treatments, illness management, rehabilitation, rheumatology 


\section{Hintergrund}

Im deutschen Sprachraum gibt es für die psychologische Behandlung von Rheumapatienten nur wenige veröffentlichte Konzepte und Forschungsergebnisse. Literatur die man findet ist zudem schon älter, so dass Überarbeitungsbedarf besteht [1,2]. Es gibt bislang keine allgemein konsentierten Empfehlungen auf Basis systematischer Literaturarbeiten. Auch von Seiten der Deutschen Rentenversicherung Bund (DRV Bund) gibt es keine rheumaspezifischen Empfehlungen. In den letzten Jahren wurden allerdings im Rahmen von Forschungsprojekten indikationsbezogene Praxisempfehlungen für psychologische Interventionen in verschiedenen anderen Indikationen entwickelt (chronischer Rückenschmerz, KHK, Schlaganfall, Diabetes mellitus und onkologische Erkrankungen [3]). Diese Praxisempfehlungen basieren auf systematischen Literaturreviews von empirischer Evidenz und Leitlinien, Befragungen zu Strukturen und Praxis in den Kliniken, Expertenworkshops, sowie Fokusgruppen mit Patienten. In Anlehnung an die vorliegenden Praxisempfehlungen werden im Folgenden unter Einbezug der Versorgungsdaten aus der externen Qualitätssicherung der DRV Bund grundsätzliche Aspekte psychologischer Interventionen in der rheumatologischen Rehabilitation skizziert.

In der internationalen Forschung erwiesen sich kognitiv-verhaltenstherapeutisch orientierte Behandlungen bei Erkrankungen aus dem rheumatischen Formenkreis als wirkungsvoll zur Symptomreduktion und Reduktion der Beeinträchtigung [4-10], z.T. auch im Langzeitverlauf über mehr als sechs Monate $[11,12]$. In deutschen Behandlungsleitlinien wurden entsprechende kognitivverhaltenstherapeutische Interventionen als evidenzbasierte Empfehlung in den AWMF-Leitlinien mit aufgenommen [13]. Multidisziplinäre Therapieansätze haben sich bei chronischen Schmerzproblemen als wirkungsvoll erwiesen und werden empfohlen [14]. Auch auf die Förderung von Selbstmanagementfertigkeiten zur aktiven Krankheitsbewältigung wird seit langem hingewiesen [5,15]. All dies sind psychologische Methoden, die auch Anwendung in der rheumatologischen Rehabilitation in Deutschland finden. 


\section{Rheumapatienten in der medizinischen Rehabilitation}

Rheumapatienten sind eine kleine Indikationsgruppe in der medizinischen Rehabilitation der Rentenversicherung, mit jedoch hoher sozialmedizinischer Relevanz. Zum rheumatologischen Formenkreis gehören entzündliche Polyarthriden (M05-M14), Systemkrankheiten des Bindegewebes (M45-M49) sowie Spondylopathien, u.a. M45 Spondylitis ankylosans (Morbus Bechterew), sowie Fibromyalgie als chronische Schmerzerkrankung des Gelenk- und Bewegungsapparates (M79.7). Von 2008 bis 2012 war insbesondere bei den Spondylopathien eine prozentuale Steigerung als Reha-Hauptdiagnose um $32 \%$ zu verzeichnen. Überdurchschnittlich viele - 28\% - der Patienten mit Systemerkrankungen des Bindegewebes haben eine schlechte Prognose in der sozialmedizinischen Beurteilung $(<6$ Stunden leistungsfähig für die letzte Tätigkeit) [16].

Rheumapatienten werden in der medizinischen Rehabilitation überwiegen in internistischrheumatologischen, zu einem geringen Anteil auch in entsprechend qualifizierten orthopädischen Abteilungen behandelt. Es gibt wenige internistische Abteilungen mit dem Schwerpunkt Rheumatologie. Für die Krankheitsbilder der Rheumatologie gibt es keine Reha-Therapiestandards der Rentenversicherung [17].

\section{Psychologische Interventionen in der rheumatologischen Rehabilitation}

\section{Aufnahme und Therapieplanung}

Die Rehabilitanden werden von ärztlicher Seite aufgenommen. Der aufnehmende Arzt führt die körperliche Untersuchung durch, erhebt die Anamnese und den aktuellen somatischen und falls notwendig den psychopathologischen Befund. Für eine begleitende Messung einer möglichenpsychischen Belastung können entsprechend der DRV-Praxisempfehlungen und Leitlinien psychologischer Screeninginstrumente verwendet werden (z.B. PHQ4, UKS, HADS [18]). Erhöhte Screeningwerte weisen dabei auf einen diagnostischen Abklärungsbedarf hin. Sie sind für sich genommen kein Beleg für eine psychische Erkrankung. Selbstverständlich können auch andere als die in dem Empfehlungen genannte Instrumente Verwendung finden. Unabhängig von standardisierten Fragebögen sind jedoch vor allem die klinische Beobachtung und Befundung und das direkte Patientengespräch von Bedeutung. 
Auf der Grundlage von Anamnese, Vorbehandlung und aktuellen klinischen Untersuchungsbefunden stimmen der aufnehmende Arzt und der Rehabilitand die Reha-Therapieziele ab. Von den RehaTherapiezielen wird der Therapieplan abgeleitet, der auch die psychologischen Therapieangebote umfasst.

In einigen Rehabilitationseinrichtungen führen die Psychologen selbst kurze Aufnahmegespräche mit allen Rehabilitanden durch. Die Indikationsstellung ob und welche psychologischen Interventionen in den Behandlungsplan aufgenommen werden, hängt von der individuellen Fragestellung und dem jeweiligen klinischen Befund ab. Die Zuweisung sollte im Behandlungsteam und in Absprache mit dem zuständigen Gesamtbehandlungsverantwortlichen getroffen werden. Beratungsanlässe können ein überdurchschnittliches psychisches Belastungserleben des Rehabilitanden sein, die Abklärung einer psychischen Erkrankung, oder Fragen der Lebens- und Alltagsbewältigung bei chronischer Erkrankung.. Eine kurze persönliche Kontaktaufnahme mit dem Psychologen zu Beginn der Rehabilitation, bspw. auch im Rahmen eines Einführungsvortrags, kann für viele Rehabilitanden die Schwelle senken, psychologische Angebote in Anspruch zu nehmen.

\section{Psychologische Einzelgespräche}

Rund ein Drittel der Rehabilitanden erhalten zumindest ein psychologisches Einzelgespräch in der Rehabilitation (Abb. 1). Im Durchschnitt werden 1-2 Gespräche geführt. Die psychologischen Einzelgespräche dienen häufig der emotionalen Entlastung und der Klärung krankheitsbezogener, aber auch familiärer oder beruflicher Probleme. Allerdings ist in einer rheumatologischen Rehabilitationsbehandlung von drei Wochen eine Psychotherapie komorbider psychischer Erkrankungen nicht möglich. Geleistet werden kann eine differentialdiagnostische Klärung und Erarbeitung einer hilfreichen Weichenstellung für die ambulante Weiterbehandlung nach der Rehabilitation. Rehabilitanden mit psychischen Erkrankungen sind teilweise auch bereits in ambulanter psychotherapeutischer Behandlung. In diesem Falle ist ggf. im Einzelgespräch mit dem Patienten und in Rücksprache mit dem ambulanten Primärbehandler zu klären, ob für die stationäre Rehabilitation ein bestimmtes Behandlungsziel vorliegt (z.B. psychologische Schmerzbewältigung). 


\section{Psychologische Gruppenangebote}

Psychologische Bewältigungsstrategien zum Thema Schmerz, Stress und Krankheitsfolgen werden in der Regel in psychologischen Gruppen vermittelt. Psychologische Gruppen sollen mit nicht mehr als 12 Rehabilitanden durchgeführt werden [18], um der individuellen Problemlage der einzelnen Teilnehmer gerecht werden zu können. Es braucht Zeit um Einstellungs- und Verhaltensänderungen anzustoßen zu können. So sollten die Gruppen mindestens drei Sitzungen à 60 Minuten umfassen. Rund ein Drittel der Rehabilitanden nehmen an einer dieser psychologischen Gruppen teil (Abb. 2). Es gibt jedoch eine nicht unbedeutsame Varianz zwischen den Kliniken hinsichtlich Umfang und Dauer der psychologischen Gruppen. So variiert beispielsweise zwischen den Kliniken die Gesamtdauer der Gruppensitzungen zwischen 3,3 und 6,7 Stunden. Um einen vertrauensvollen Austausch zu befördern, wird empfohlen „geschlossene“ Gruppen durchzuführen. Die einzelnen Sitzungen haben einzelne Themenschwerpunkte, die je nach Gruppe den Bedürfnissen der Teilnehmer angepasst werden. Ein älteres psychologisches Gruppenkonzept für die Rheumatologie [1] beinhaltete Themen wie Schmerz und Krankheitsverständnis, Kognitionstechniken / Schmerzobjektivierung, Genusstraining, Soziale Kompetenz, Verlust und Trauer. Als Ziele der Schulung werden Wissenserwerb, Training von Fertigkeiten, Unterstützung zur Reduktion von Angst und Depressivität und das Training der krankheitsbezogenen sozialen Kompetenz beschrieben. Psychologische Schmerzbewältigungstrainings haben in der rheumatologischen Rehabilitation einen hohen Stellenwert und zielen darauf ab, Erlebens- und Verhaltensaspekte bei Schmerzen zu verändern.

\section{Entspannungstraining}

Das Entspannungstraining wird in der rheumatologischen Rehabilitation von Psychologen oder auch anderen dafür qualifizierten Berufsgruppen, bspw. Bewegungstherapeuten, durchgeführt. Die Einführung in die verschiedenen Entspannungsverfahren mit ihrer besonderen Bedeutung für die Schmerzwahrnehmung und - bewältigung führen regelhaft die Psychologen durch. Bei übenden Verfahren wie der Progressiven Muskelrelaxation wird das Training selbst häufig von entsprechend fortgebildeten Physiotherapeuten und anderen Berufsgruppen der medizinischen Rehabilitation angeboten. Stehen psychologische Aspekte der Entspannungsfähigkeit im Vordergrund, wird das Entspan- 
nungstraining häufig auch in die psychologischen Schmerzbewältigungsgruppen integriert. Das Entspannungstraining wird entsprechend des Katalogs Therapeutischer Leistungen der DRV (KTL [19]) unterschiedlich verschlüsselt, entweder als Übungseinheit, als Einführungsveranstaltung oder als Teil der psychologischen Gruppe. Möglicherweise erklären konzeptionelle Unterschiede auch die große Varianz zwischen den Rehabilitationseinrichtungen. So liegt die Inanspruchnahme von Leistungen des Entspannungstrainings außerhalb einer psychologischen Gruppe zwischen $42 \%$ und $89 \%$, die Gesamtdauer des Trainings streut dabei zwischen 2,6 und 3,6 Stunden (Abbildung 3). Der Erfolg dieser und anderer übungsorientierter Behandlungsmaßnahmen ist zu einem maßgeblichen Anteil auch von der Übungs-Compliance der betreuten Patienten abhängig. Daher spielt auch die Motivationsklärung und -förderung eine wichtige Rolle in der Betreuung der Patienten.

\section{Intensivierte psychologische Behandlung}

Ein spezifisches Rehabilitationskonzept für Rehabilitanden mit psychischer Komorbidität ist die verhaltensmedizinisch orientierte Rehabilitation (VOR [20], vgl. Dorn in diesem Heft). In der verhaltensmedizinisch orientierten Rehabilitation wird ein höherer Stellenschlüssel im Bereich Psychologie vorgehalten $(1: 25)$. Die Psychologen sind approbiert und können auch psychotherapeutische und differentialdiagnostische Leistungen durchführen. Psychologische Gruppenprogramme der VOR (8-12 Patienten in einer Gruppe) nutzen den intensiven Austausch in einer festen Bezugsgruppe, um u.a. ein bio-psycho-soziales Verständnis vom Schmerzerleben zu erarbeiten.

\section{Patientenschulungen und -vorträge}

Psychologen sind in der rheumatologischen Rehabilitation auch an edukativen Leistungen beteiligt. Dies betrifft sowohl Patientenvorträge zu spezifischen psychologischen Themen wie Stress und Stressbewältigung als auch die interdisziplinär konzipierte Patientenschulung. Patientenschulungen in der rheumatologischen Rehabilitation haben einen sehr hohen Stellenwert. Sie umfassen Informationen zu Ursachen und Verläufen von Erkrankungen, Behandlungsmöglichkeiten und die Anleitung zum krankheitsbezogenen Selbstmanagement Die ersten rheumatologischen Schulungsprogramme wurden bereits in den 90iger Jahren von der Deutschen Gesellschaft für Rheumato- 
logie für die Indikationen chronischer Polyarthritis, Morbus Bechterew und Lupus erythematodes entwickelt. Weitere Schulungsprogramme folgten für die Indikationen Fibromyalgie, Osteoporose, sowie für rheumakranke Kinder und deren Eltern [10, 21,22].

Evaluierte Schulungskonzepte sind verfügbar für Patienten mit Fibromyalgie [10], chronischer Polyarthritis [23], sowie Patienten mit Spondylitis ankylosans [24].

Psychologisches Thema ist in den Schulungen u.a. eine Einführung in das psychologische Verständnis von Schmerz. Aber auch soziale Kompetenzaspekte und das Thema Selbsthilfe werden von psychologischer Seite in Patientenschulungen der rheumatologischen Rehabilitation aufgegriffen. Diese Aspekte sind z.B. wichtig für die Kommunikation mit Ärzten, Angehörigen und Kollegen bzw. Arbeitgebern. Ein wichtiges Ziel ist auch die Förderung der Compliance bzgl. einer ärztlich kontrollierten Schmerzmitteleinnahme, sowie die Reduktion von Selbstmedikation und unkontrollierter Einnahme von Schmerzmitteln nach Bedarf.

Patientenschulungen zur Krankheitsinformation und Krankheitsbewältigung sollten die Schulungsinhalte in interdisziplinärem Vorgehen vermitteln [2]. Es sollte ein Schulungskonzept, Curriculum oder Manual vorliegen, in dem die wichtigsten zu vermittelnden Inhalte zusammengefasst sind. Die Arbeit in Kleingruppen hat sich als hilfreich erwiesen, um den Austausch der Rehabilitanden untereinander zu fördern.

\section{Berufsbezogene Interventionen}

Die Wiederherstellung oder der Erhalt der Erwerbsfähigkeit ist die zentrale Aufgabe der medizinischen Rehabilitation der Rentenversicherung. Berufsorientierte Konzepte wurden in den letzten Jahren verstärkt ausgebaut (Anforderungsprofil Medizinisch-beruflich-orientierte Rehabilitation, MBOR [25]). Auch von psychologischer Seite werden Gruppenangebote zu den Themen arbeitsbezogene Stressbewältigung [26,27], Zeitmanagement, Arbeitsplatzkonflikte und der Umgang mit Ängsten und körperlichen Beeinträchtigungen bei der Arbeit [28] angeboten. Weiterentwicklungen der beruflichorientierten Rehabilitation sind vonnöten hinsichtlich der direkten Kommunikation mit den Arbeitgebern für die Herstellung leidensgerechter Arbeitsplätze, und einer auf Verständnis abzielenden Klärung der überdauernden Beeinträchtigungen und des Umgangs damit am Arbeitsplatz. 


\section{Psychodiagnostik und Einschätzung der psychischen Funktionsfähigkeit}

Um zu klären, ob ein Rehabilitand eine psychische Erkrankung hat, ist eine klinische Differentialdiagnostik durch einen Psychologischen Psychotherapeuten und/oder einen entsprechend qualifizierten Arzt notwendig. Sämtliche standardisierte psychologische Testverfahren [18], die ggf. als ergänzende Verlaufsdiagnostik genutzt werden, können dazu dienen um mit dem Patienten zu diesen Themen ins Gespräch zu kommen, sie ersetzen nicht die klinische Diagnostik [29].

Psychisches Belastungserleben nicht-krankheitswertiger Art kann als Z-Diagnose dokumentiert werden und wird dabei von psychischen Erkrankungen (F-Diagnosen) im engeren Sinne abgegrenzt [30]. Im Zweifelsfalle kann ein psychosomatisches oder psychiatrisches Konsil hinzu gezogen werden. Für die sozialmedizinische Leistungsbeurteilung am Ende der Rehabilitation sind alle Befunde aus dem Reha-Team von Bedeutung [31]. Bei einzelnen Rehabilitanden kommt Psychologen die Aufgabe zu, einen Beobachtungsbefund des psychischen Fähigkeits- und Aktivitätsniveaus darzulegen [32]. In der rheumatologischen Rehabilitation betrifft dies ausschließlich die Frage der aktuellen Arbeitsfähigkeit. Ist prognostisch anzunehmen, dass die psychischen Fähigkeitsbeeinträchtigungen chronisch sind und zu einer Minderung der Erwerbsfähigkeit führen, muss im Anschluss an die rheumatologische Rehabilitation eine psychiatrische Begutachtung stattfinden.

\section{Teamarbeit und Berichterstellung}

Fallbezogene Teamsitzungen sind ein wichtiges Qualitätsmerkmal in der medizinischen Rehabilitation. An den Teamsitzungen sollten alle an der Behandlung beteiligten Berufsgruppen teilnehmen [33]. Jede Berufsgruppe kann mit wichtigen Informationen aus dem Behandlungsverlauf zur sozialmedizinischen Leistungsbeurteilung beitragen. Von Beginn des Rehabilitationsaufenthaltes an können Therapeuten im Reha-Team mit konkreten Beobachtungszielen im Sinne des Gesamtbehandlungsplanes ihre Behandlungen gestalten (z.B. Bewegungstherapeut: Training der körperlichen Ausdauerleistung, Ergotherapeut: Konzentrationsfähigkeit, Handfunktion). Voraussetzung ist, dass ein Gesamtbehandlungsziel zur Beginn des Rehabilitationsaufenthaltes im Team abgestimmt und klar formuliert wird (z.B. Verbesserung der Durchhaltefähigkeit). 
Bei Rehabilitanden mit besonderen Problemlagen, insbesondere bei Vorliegen einer psychischen Komorbidität oder überdauernder krankheitsbedingter Fähigkeitsbeeinträchtigung, die bedeutsam für die sozialmedizinische Leistungseinschätzung ist, schreiben die Psychologen einen internen Bericht. Der psychologische Bericht sollte die im konkreten Einzelfall relevanten Fakten beschreiben [34], insbesondere die zuweisende Fragestellung und den konkreten Behandlungsauftrag für die Rehabilitation, die entsprechende Diagnostik (z.B. Verhaltensbeobachtungen, Übungssituationen, kognitive Tests), sowie Informationen für die sozialmedizinische Beurteilung. Hierbei geht es um die Leistung und Leistungsfähigkeit, d.h. was der Rehabilitand aus der Beobachtung in der Klinik heraus kann und was er unter bestimmten Arbeitsbedingungen tun könnte. Im Reha-Entlassungsbericht werden im Abschnitt der sozialmedizinischen Leistungsbeurteilung die erkrankungsbedingten Aktivitäts- und Fähigkeitsbeeinträchtigungen beschrieben, die die Erwerbsfähigkeitsprognose begründen.

\section{Alltagstransfer und Nachsorgeleistungen}

Im Hinblick auf die Nachhaltigkeit der Verhaltensänderung sind während der stationären Rehabilitation bereits Bewältigungsmöglichkeiten für den Alltag zu planen, bspw. der Besuch weiterführender Bewegungsgruppen oder Selbsthilfeangebote (www.rheuma-liga.de) am Heimatort, aber auch individuelle Bewältigungsstrategien für die alltäglichen Aufgaben u.ä..

Bei Rehabilitanden mit psychischer Komorbidität ist abzuklären, ob eine Indikation für psychotherapeutische Maßnahmen im engeren Sinne besteht. Es gibt viele verschiedene Varianten, wie Rehabilitanden auch nach dem Rehaaufenthalt erfolgreich psychotherapeutisch oder supportiv begleitet werden können. Dies schließt auch eine hausärztliche Behandlung im Rahmen der psychosomatischen Grundversorgung mit ein. Eine konkrete Empfehlung sollte im ärztlichen Entlassungsbericht benannt werden.

\section{Ausblick}

Psychologische Interventionen sind ein fester Bestandteil in der rheumatologischen Rehabilitation der Deutschen Rentenversicherung. Die Rentenversicherung unterstützt die Qualifikation von Therapeuten im Reha-Team (Diplom-Psychologen, Ergotherapeuten, Sozialarbeiter u.a.) im Hinblick auf die Anfer- 
tigung guter Fähigkeits- und Verhaltensbeobachtungen und -beschreibungen. Die Befunde der Therapeuten haben im Rahmen der sozialmedizinischen Leistungsbeurteilung am Ende der Rehabilitation einen hohen Stellenwert.

Zum Zwecke einer fachkundigen Differentialdiagnostik psychischer Komorbidität sollten perspektivisch verstärkt approbierte Psychologen in der rheumatologischen Rehabilitation beschäftigt werden. Aus den DRV-Qualitätsdaten wird erkennbar, dass bei gleichen Strukturvorgaben in verschiedenen Kliniken psychologische Interventionen in unterschiedlicher Weise umgesetzt werden. Die Umsetzung von formalen Strukturvorgaben ist dabei sicherlich immer auch ein Abwägen zwischen Priorisierung einer guten Patientenversorgung, fachlicher Machbarkeit und Normenerfüllung. Begrüßt wird von psychologischer Seite, dass gesundheitspsychologische Ansätze der Handlungs- und Bewältigungsplanung bei einer Überarbeitung der Patientenschulungskonzepte der DGRh Berücksichtigung finden [2]. 


\section{Literatur}

[1] Jungnitsch G. Psychologische Interventionen für rheumatische Erkrankungen. Psychomed 1991;3:181-184

[2] Faller H, Ehlebracht-König I, Reusch A. Empowerment durch Patientenschulung in der Rheumatologie. Zeitschrift für Rheumatologie 2015;74:603-608

[3] Reese C, Mittag O. Psychologische Interventionen in der Rehabilitation von Patienten mit onkologischen Erkrankungen: Systematische Entwicklung von Praxisempfehlungen. DRV-Schriften 2016;109:400-401

[4] Dixon KE, Keefe FJ, Scipio CD, et al. Psychological interventions for arthritis pain management in adults: a meta-analysis. Health Psychology 2007;26:241-250

[5] Knittle K, Maes $S$, de Gucht $V$. Psychological interventions for rheumatoid arthritis: examining the role of self-regulation with a systematic review and meta-analysis of randomized controlled trials. Arthritis Care Research 2010;62:1460-1472

[6] Astin JA, Beckner W, Soeken K. Psychological interventions for rheumatoid arthritis: a metaanalysis of randomized controlled trials. Arthritis Rheum 2002;15:291-302

[7] Savelkoul M, de Witte L, Post M. Stimulating active coping in patients with rheumatic diseaeses : a systematic review of controlled group intervention studies. Patient Educ Couns 2003;50:133-143

[8] Sharpe L, Sensky T, Timberlake N, et al. Long-term efficacy of a cognitive behavioral treatment from a randomized controlled trial for patients recently diagnosed with rheumatoid arthritis. Rheumatology 2003;42:435-441

[9] Sharpe L, Allard S, Sensky T. Five-year follow-up of a cognitive-behavioral intervention for patients with recently-diagnosed rheumatoid arthritis: effects on health care utilization. Arthritis Rheum 2008;59:311-316

[10] Siemienik K, Reusch A, Musekamp G. Ein Schulungsprogramm für Patienten und Patientinnen mit Fibromyalgie-Syndrom. Manual. Online abgerufen am 12.02.2016. Verfügbar unter http://www.zentrum-patientenschulung.de/forschung/projekte/FimS/FimS_Manual.pdf

[11] Bernardy K, Klose P, Busch AJ. Cognitive behavioural therapies for fibromyalgia. Cochrane Database Systematic Reviews 2013; 9:CD009796. doi: 10.1002/14651858.CD009796.pub2.

[12] Evers $A W$, Kraaimaat $F W$, van Riel $P L$, et al. Tailored cognitive-behavioral therapy in early rheumatoid arthritis for patients at risk: a randomized controlled trial. Pain 2002;100:141-153

[13] AWMF. Definition, Pathophysiologie, Diagnostik und Therapie des Fibromyalgiesyndroms 041/004. S3-Leitlinie. Arbeitsgemeinschaft der Wissenschaftlichen medizinischen Fachgesellschaften e.V., 2012

[14] Scascighini L, Toma V, Dober-Spielmann S, et al. Multidisciplinary treatment for chronic pain: a systematic review of interventions and outcomes. Rheumatology 2008;47:670-678

[15] Moffet J, McLean S. The role of physiotherapy in the management of non-specific back pain and neck pain. Rheumatology 2006;45:371-378 
[16] Egner U, Kalwa M, Jenner B, et al. Anforderungen an die Rheuma-Reha aus Sicht eines Leistungsträgers. Düsseldorf: Vortrag auf dem 42. Kongress der Deutschen Gesellschaft für Rheumatologie (DGRh), 2014

[17] DRV Deutsche Rentenversicherung Bund. Reha-Therapiestandards für die medizinische Rehabilitation der Rentenversicherung. Berlin: DRV Bund, 2016, online abrufbar unter http://www.deutscherentenversiche-

rung.de/Allgemein/de/Navigation/3_Infos_fuer_Experten/01_Sozialmedizin_Forschung/02_reha_qual itaetssicherung/reha_therapiestandards/indikationen_index_node.html.

[18] DRV Deutsche Rentenversicherung Bund. Reader Psychologie. Aktuelle Informationen zur psychologischen Arbeit in der medizinischen Rehabilitation. Berlin: Deutsche Rentenversicherung Bund, 2015.

[19] DRV Deutsche Rentenversicherung Bund. Klassifikation therapeutischer Leistungen in der medizinischen Rehabilitation. Berlin: DRV Bund, 2015

[20] DRV Deutsche Rentenversicherung Bund. Verhaltensmedizinisch orientierte Rehabilitation. Berlin: DRV Bund, 2014.

[21] Ehlebracht-König I. Patientenschulung in der Rheumatologie. Zeitschrift für Rheumatologie 2003;62:II/6-II/9

[22] Ehlebracht-König I, Bönisch A. Patientenschulung im frühen Krankheitsmanagement der Spondylitis ankylosans und verwandter Spondyloarthriden. Wiener Medizinische Wochenschrift 2008, 158/78:213-217

[23] Langer HE, Ehlebracht-König I, et al. Evaluation des Patientenschulungskurses "Chronische Polyarthritis". Deutsche Rentenversicherung 1998;4:239-264

[24] Bönisch A, Ehlebracht-König I, Krauth C, et al. Evaluation eines Schulungsseminares für Patienten mit Spondylitis ankylosans (SpA). In: Petermann F (Hrsg) Prädiktion, Verfahrensoptimierung und Kosten in der medizinischen Rehabilitation. 2. Aufl . Regensburg: Roderer, 2005;51-102

[25] DRV Deutsche Rentenversicherung Bund. Medizinisch-beruflich-orientierte Rehabilitation Anforderungsprofil zur Durchführung der medizinisch-beruflich-orientierten Rehabilitation im Auftrag der Deutschen Rentenversicherung. Berlin: DRV Bund, 2015

[26] Bethge M, Neuderth S. Medizinisch-berufliche Maßnahmen. In: Bengel J, Mittag O (Hrsg). Psychologie in der medizinischen Rehabilitation. Berlin: Springer, 2016, 149-195

[27] Rotter M, Renneberg B, Kaluza G. Stressbewältigung. In: Bengel J, Mittag O (Hrsg). Psychologie in der medizinischen Rehabilitation. Berlin: Springer, 2016, 161-172

[28] Muschalla B, Fay D, Jöbges M, et al. Evaluation einer Gruppentherapie für arbeitsplatzbezogene Ängste und Arbeitsplatzphobie. Behandlungsmanual und Abschlussbericht zum DRV-

Forschungsprojekt. Brandenburgklinik Bernau und Universität Potsdam, Arbeits- und Organisationspsychologie, 2014

[29] Muschalla B, Linden M. Standardized diagnostic interviews, criteria, and algorithms for mental disorders: garbage in, garbage out. Eur Arch Psychiatry Clin Neurosci 2012;262:535-544

[30] WHO World Health Organization. International Statistical Classification of Diseases and Related Health Problems, $10^{\text {th }}$ revision. Geneva: World Health Organization, 1992 
[31] DRV Deutsche Rentenversicherung Bund. Curriculum Sozialmedizinische Leistungsbeurteilung im Reha-Team. Berlin: DRV Bund, 2015

[32] Linden M, Baron S, Muschalla B et al. Fähigkeitsbeeinträchtigungen bei psychischen Erkrankungen. Diagnostik, Therapie und sozialmedizinische Beurteilung in Anlehnung an das Mini-ICF-APP. Göttingen: Hogrefe, 2015

[33] Körner M Dorn M. Reha-Team und Teamentwicklung. In: Bengel J, Mittag O (Hrsg). Psychologie in der medizinischen Rehabilitation. Berlin: Springer, 2016, 239-249

[34] Worringen U, Kleinhans M, Schmucker D. Psychologischer Bericht und Reha-Entlassungsbericht. In: Bengel J, Mittag O (Hrsg). Psychologie in der medizinischen Rehabilitation. Berlin: Springer, 2016, 83-92

Abbildung 1: Psychologische Gespräche in Reha-Kliniken

Abbildung 2: Psychologische Gruppen in Reha-Kliniken

Abbildung 3: Entspannungstraining in Reha-Kliniken 